\title{
Harmonic Balance Method for Chaotic Dynamics in Fractional-Order Rössler Toroidal System
}

\author{
Huijian Zhu ${ }^{1,2}$ \\ ${ }^{1}$ School of Sciences, South China University of Technology, Guangzhou 510640, China \\ ${ }^{2}$ School of Electromechanics, Guangdong Polytechnic Normal University, Guangzhou 510635, China \\ Correspondence should be addressed to Huijian Zhu; hjzhu2013@gmail.com
}

Received 23 May 2013; Accepted 8 November 2013

Academic Editor: Recai Kilic

Copyright (c) 2013 Huijian Zhu. This is an open access article distributed under the Creative Commons Attribution License, which permits unrestricted use, distribution, and reproduction in any medium, provided the original work is properly cited.

\begin{abstract}
This paper deals with the problem of determining the conditions under which fractional order Rössler toroidal system can give rise to chaotic behavior. Based on the harmonic balance method, four detailed steps are presented for predicting the existence and the location of chaotic motions. Numerical simulations are performed to verify the theoretical analysis by straightforward computations.
\end{abstract}

\section{Introduction}

The concept of fractional calculus has been known since the development of the regular calculus, with the first reference probably being associated with Leibniz and L'Hôspital in 1695. In the past three decades or so, fractional calculus gained considerable popularity and importance, due mainly to its demonstrated applications in numerous seemingly diverse and widespread fields of science and engineering [13]. In particular, by utilizing fractional calculus technique, many investigations were devoted to the chaotic behaviors and chaotic control of dynamical systems involving the fractional derivative, called fractional-order chaotic system [4-8]. For example, it has been shown that Chua circuit of the order as low as 2.7 can behave in chaotic manner [4]. In [5], the nonautonomous Duffing systems of the order less than 2 can still produce a chaotic attractor. In [6], chaotic behavior of the fractional-order Lorenz system was further studied. In [7], chaos and hyperchaos in the fractional-order Rössler equations were also studied, in which the authors showed that chaos can exist in the fractional-order Rössler equation with the order as low as 2.4 , and hyperchaos can exist in the fractional-order Rössler hyperchaos equation with the order as low as 3.8. Later on, the chaotic behavior and its control in the fractional-order Chen system were investigated in [8].
And recently, more dynamic behaviors of fractional order chaotic systems were analyzed by using different approaches; we refer the readers to [9-12].

However, to our knowledge, the conditions for chaos existence in dynamical systems (including integer-order system and fractional-order system) are still incomplete. For a given dynamical system, can we decide (without invoking numerical simulations) whether and in what parameter ranges, chaotic behavior might exist? It is still an open problem [13]. Even though some theorems such as Melnikov's criteria [14] and Shil'nikov's theorem [15] may be helpful in some special cases, it seems that a powerful tool is not generally available to determine the accurate parameter ranges for chaos existence in a given dynamical system. Moreover, the fact that fractional-order system possess long memory characteristics makes the system behave in more complicated manner. This means that chaos analysis in fractional-order systems is more complicated than that in integer-order systems. Recently, based on harmonic balance method (or describing function method), predicting chaotic dynamics in fractionalorder Genesio-Tesi system was investigated [16]. Later on, two describing function-based techniques were proposed to predict chaos in a class of fractional-order differential equations [17]. Therefore, harmonic balance method can give another way to detect the conditions for chaos existence 
in fractional-order systems. Following them, the subject of this paper is predicting conditions for chaos existence in fractional-order Rössler toroidal system.

The paper is organized as follows. In Section 2, some basic concepts of fractional calculus are briefly introduced. Sections 3 and 4 are about predicting chaos in fractionalorder Rössler toroidal system by utilizing harmonic balance method. In Section 5, numerical simulations are further presented to confirm the obtained results of Section 4. Finally, the brief conclusions are drawn in Section 6 and close the paper.

\section{Preliminaries}

2.1. Basic Definitions. In what follows, the definitions of fractional derivative are introduced in brief. For an arbitrary real number $q$, the Riemann-Liouville and Caputo fractional derivatives are defined, respectively, as

$$
\begin{gathered}
{ }_{a} D_{t}^{q} f(t)=\frac{1}{\Gamma(m-q)} \frac{d^{m}}{d t^{m}} \int_{a}^{t}(t-\tau)^{m-q-1} f(\tau) d \tau \\
\quad(m=[q]+1), \\
{ }_{a}^{C} D_{t}^{q} f(t)=\frac{1}{\Gamma(m-q)} \int_{a}^{t} \frac{f^{(m)}(\tau)}{(t-\tau)^{q+1-m}} d \tau \\
(m=[q]+1),
\end{gathered}
$$

where $[q]$ stands for the integer part of $q$; that is, $m$ is the first integer which is not less than $q$.

Note that the main advantage of Caputo approach is that the initial conditions for fractional differential equations with Caputo derivatives take on the same form as for integer-order differential equations. Some other important properties of the fractional derivatives and integrals can be found in [1-3].

2.2. Numerical Method. According to [18, 19], the predictorcorrector scheme for fractional-order system is derived. This scheme is the generalization of Adams-Bashforth-Moulton scheme. First, the following fractional-order differential equation involving Caputo derivative

$$
\begin{gathered}
\frac{d^{q} x}{d t^{q}}=f(t, x), \quad 0 \leq t \leq T, \\
x^{(k)}(0)=x_{0}^{(k)}, \quad k=0,1,2, \ldots, m-1,
\end{gathered}
$$

is equivalent to the Volterra integral equation (see [18, Lemma 2.1])

$$
x=\sum_{k=0}^{\lceil q\rceil-1} x_{0}^{(k)} \frac{t^{k}}{k !}+\frac{1}{\Gamma(q)} \int_{0}^{t} \frac{f(\tau, x)}{(t-\tau)^{1-q}} d \tau .
$$

Set $h=T / N, t_{n}=n h, n=0,1,2, \ldots, N$, and system (4) can be discretized as

$$
\begin{aligned}
x_{h}\left(t_{n+1}\right)= & \sum_{k=0}^{\lceil q\rceil-1} x_{0}^{(k)} \frac{t_{n+1}^{k}}{k !}+\frac{h^{q}}{\Gamma(q+2)} f\left(t_{n+1}, x_{h}^{p}\left(t_{n+1}\right)\right) \\
& +\frac{h^{q}}{\Gamma(q+2)} \sum_{j=0}^{n} a_{j, n+1} f\left(t_{j}, x_{h}\left(t_{j}\right)\right),
\end{aligned}
$$

where

$$
\begin{aligned}
& a_{j, n+1} \\
& =\left\{\begin{array}{l}
n^{q+1}-(n-q)(n+1)^{q}, \quad j=0, \\
(n-j+2)^{q+1}+(n-j)^{q+1}-2(n-j+1)^{q+1}, \\
1, \quad j=n+1,
\end{array}\right. \\
& x_{h}^{p}\left(t_{n+1}\right)=\sum_{k=0}^{\lceil q\rceil-1} x_{0}^{(k)} \frac{t_{n+1}^{k}}{k !}+\frac{1}{\Gamma(q)} \sum_{j=0}^{n} b_{j, n+1} f\left(t_{j}, x_{h}\left(t_{j}\right)\right), \\
& b_{j, n+1}=\frac{h^{q}}{q}\left((n+1-j)^{q}-(n-j)^{q}\right) .
\end{aligned}
$$

The error estimate is $e=\max \left|x\left(t_{j}\right)-x_{h}\left(t_{j}\right)\right|=O\left(h^{p}\right)(j=$ $0,1,2, \ldots, N)$, in which $p=\min (2,1+q)$.

\section{Fractional Rössler Toroidal System}

The Rössler toroidal system, which was firstly introduced in [20], is defined by

$$
\begin{gathered}
\frac{d x}{d t}=-y-z, \\
\frac{d y}{d t}=x, \\
\frac{d z}{d t}=a y-a y^{2}-b z,
\end{gathered}
$$

where $a>0$ and $b>0$ are real parameters.

For what follows, it is useful to transform this system in the equivalent third-order differential equation:

$$
\frac{d^{3} y}{d t^{3}}+b \frac{d^{2} y}{d t^{2}}+\frac{d y}{d \mathrm{t}}+(a+b) y-a y^{2}=0 .
$$

Now we introduce fractional order into the system (8) and obtain the corresponding fractional-order Rössler toroidal model:

$$
\frac{d^{2+q} y}{d t^{2+q}}+b \frac{d^{2} y}{d t^{2}}+\frac{d y}{d t}+(a+b) y-a y^{2}=0
$$

where $a>0, b>0,0<q<1$, and $d^{q} / d t^{q}$ is in sense of the Caputo fractional derivative defined in (2) with the initial time $t=0$. 
According to the Poincare-Bendixson theorem [21], if $q$ is very close to zero, then system (9) behaves like a two-dimensional system and cannot be chaotic. However, if $q$ tends to 1 , system (9) dynamically approaches a thirdorder ordinary differential equation which can be chaotic. So, it is interesting to analyze the conditions under which chaos can occur in system (9) when $q \in(0,1)$.

First, system (9) can be represented as a nonlinear feedback structure, made of a linear dynamic part $G$ and a nonlinear dynamic part $N$ (shown in Figure 1).

The linear subsystem $G$ is modeled by the following transfer function:

$$
G(s)=\frac{1}{s^{2+q}+b s^{2}+s+a+b},
$$

and the nonlinear subsystem $N$ is described by a real function

$$
f(y)=-a y^{2} .
$$

Suppose that the hypothetical solution of system (9) is represented by

$$
y_{0}(t)=A+B \sin \omega t,
$$

where $B>0$ and $\omega>0$. Also, the nonlinear subsystem $f\left(y_{0}\right)$ can be approximated by

$$
f\left(y_{0}\right) \approx N_{0}(A, B) A+N_{1}(A, B) B \sin \omega t,
$$

where $N_{0}$ and $N_{1}$ are represented by

$$
\begin{aligned}
N_{0}(A, B) & =\frac{1}{2 \pi A} \int_{-\pi}^{\pi} f(A+B \sin \omega t) d \omega t \\
& =-a A-\frac{a B^{2}}{2 A}, \\
N_{1}(A, B)= & \frac{1}{\pi B} \int_{-\pi}^{\pi} f(A+B \sin \omega t) \sin \omega t d \omega t=-2 a A .
\end{aligned}
$$

Note that this approximation can be satisfied, if the linear part $G(j \omega)$ has the property of a low pass filter. From Figure 1, it follows

$$
\begin{gathered}
-\left(G(0) N_{0}(A, B) A+G(j \omega) N_{1}(A, B) B \sin \omega t\right) \\
=A+B \sin \omega t .
\end{gathered}
$$

Thus, the following equations are obtained:

$$
\begin{gathered}
A\left(1+G(0) N_{0}(A, B)\right)=0, \\
1+G(j \omega) N_{1}(A, B)=0 .
\end{gathered}
$$

\section{The Conditions for Chaos to Occur}

Based on the Genesio-Tesi conjecture [22], the predicted existence of chaos can be synthesized, with the following four conditions satisfied: (i) existence of a stable limit cycle

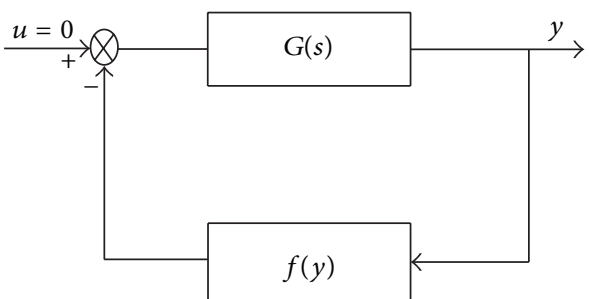

FIGURE 1: Nonlinear feedback structure of the system (9).

$y_{0}(t)=A+B \sin \omega t$, (ii) existence of an unstable equilibrium point $E$ which is different from the fixed point that originates the predicted limit cycle in (i), (iii) an interaction between the observed limit cycle $y_{0}(t)$ and equilibrium point $E$; that is, $y_{0}(t)=E$ for some $t$, (iv) suitable filtering effect in the Genesio-Tesi conjecture. So, the following four steps must be performed.

4.1. A Stable Limit Cycle. Suppose that the predicted limit cycle is denoted by $y_{0}(t)=A_{0}+B_{0} \sin \omega_{0} t$. Substituting (15) into (18) and letting the real and imaginary parts be equal to zero, respectively, their solutions can be expressed as follows:

$$
\begin{gathered}
\omega_{0}=\left(\frac{1}{\sin (q \pi / 2)}\right)^{1 /(1+q)}, \\
A_{0}=\frac{1}{2 a}\left[-\left(\frac{1}{\sin (q \pi / 2)}\right)^{(2+q) /(1+q)} \cos \left(\frac{q \pi}{2}\right)\right. \\
\left.-b \sin \left(\frac{q \pi}{2}\right)^{2 /(1+q)}+a+b\right] .
\end{gathered}
$$

From (14) and (17), it follows

$$
B_{0}=\sqrt{\frac{2(a+b) A_{0}-2 a A_{0}^{2}}{a}},
$$

which implies that

$$
A_{0}\left(a+b-a A_{0}\right)>0 .
$$

Since $a>0$ and $b>0$, the necessary condition [17] for existence of a limit cycle is

$$
\begin{aligned}
& -\left(\frac{1}{\sin (q \pi / 2)}\right)^{(2+q) /(1+q)} \cos \left(\frac{q \pi}{2}\right) \\
& -b\left(\frac{1}{\sin (q \pi / 2)}\right)^{2 /(1+q)}+a+b>0 .
\end{aligned}
$$

Based on the approximate Loeb criterion [23], the stability of the predicted limit cycle depends on the relative location of $G(j \omega)$ with respect to $\omega$ and the locus of $1 / N_{1}(A(B), B)$ with respect to $B$, where $A$ is considered as a function of $B$, that is, $A(B)$. The predicted limit cycle is stable if the locus of $1 / N_{1}(A(B), B)$ enters in a stable interval 
of the real axis by increasing $B$ from $B_{0}$ and, vice versa, by decreasing $B$ (see Figure 6 in [24]). From [17], it is sufficient to check $d N_{1}(A(B), B) /\left.d B\right|_{B=B_{0}}<0$.

According to (14)-(18), it follows

$$
\begin{aligned}
N_{1}(A(B), B) \\
=-\frac{2 a^{2} B^{2}}{(2 / G(0))-(1 / G(j \omega))} \\
=-\left(2 a^{2} B^{2}\right) \\
\quad \times\left(2(a+b)-\left[-\omega^{2+q}\left(\cos \left(\frac{q \pi}{2}\right)+j \sin \left(\frac{q \pi}{2}\right)\right)\right.\right. \\
\left.\left.\quad-b \omega^{2}+j \omega+a+b\right]\right)^{-1} .
\end{aligned}
$$

Thus, from inequalities (22) and (23), it can be calculated that

$$
\begin{aligned}
& \left.\frac{d N_{1}(A(B), B)}{d B}\right|_{B=B_{0}} \\
& =-\left(4 a^{2} B_{0}\right) \\
& \quad \times\left(2(a+b)-\left[-\left(\frac{1}{\sin (q \pi / 2)}\right)^{(2+q) /(1+q)} \cos \left(\frac{q \pi}{2}\right)\right.\right. \\
& =-\frac{2 a^{2} B_{0}}{a+b-a A_{0}} \\
& <0 .
\end{aligned}
$$

Therefore, the predicted limit cycle is stable according to the Loeb criterion [23].

4.2. An Unstable Equilibrium Point. By solving the equation $(a+b) y-a y^{2}=0$, the equilibrium points of system (9) can be derived as follows:

$$
y_{1}=0, \quad y_{2}=\frac{(a+b)}{a} .
$$

The equilibrium point $y_{2}=(a+b) / a$ is the equilibrium point of interest in this step of the described function method because the equilibrium point $y_{1}=0$ originates the limit cycle described by (19)-(21). Moreover, the characteristic equation of linearized system of the system (9) around the equilibrium point $y_{2}=(a+b) / a$ is given by

$$
P(\lambda)=\lambda^{2+q}+b \lambda^{2}+\lambda-a-b .
$$

Since $a>0$ and $b>0$, it is easy to check that $P(0)<0$ and $P(a+b)>0$. This means that there at least exists a positive real number $\lambda^{*}$ between 0 and $a+b$, which satisfies $P\left(\lambda^{*}\right)=0$. Hence, the equilibrium point $y_{2}$ is unstable according to the stability theorem proved in [25].
4.3. Interaction between the Limit Cycle and Equilibria. In this section, an interaction between the observed limit cycle $y_{0}(t)$ and equilibrium point $y_{2}$ should be verified. This condition can be restated by the following inequality:

$$
B_{0} \geq\left|y_{2}-A_{0}\right| \text {. }
$$

Because of heuristic nature of the method, condition (28) should be considered in the more indefinite form such as

$$
B_{0} \geq \theta\left|y_{2}-A_{0}\right|
$$

where $\theta$ is a number close to 1 [22]. According to (20) and (21), condition (29) can be verified if

$$
\begin{aligned}
\left(\frac{2+\theta^{2}}{2-\theta^{2}}\right)[ & -\left(\frac{1}{\sin (q \pi / 2)}\right)^{(2+q) /(1+q)} \cos \left(\frac{q \pi}{2}\right) \\
& \left.-b\left(\frac{1}{\sin (q \pi / 2)}\right)^{2 /(1+q)}\right]+a+b>0,
\end{aligned}
$$

which is stronger than the necessary condition (23). Hence, an interaction between the observed limit cycle $y_{0}(t)$ and equilibrium point $y_{2}$ is certain.

4.4. Filter Effect. Since the reliability of the stable limit cycle is based on the filtering hypothesis, the higher harmonic terms of $f(A+B \sin \omega t)$ are discarded. The amount of the neglected higher harmonics concerning the predicted limit cycle with $y_{0}(t)$ of frequency $\omega$ can be expressed by the socalled distortion index

$$
\Delta=\frac{\left\|\tilde{y}_{0}(t)-y_{0}(t)\right\|_{2}}{\left\|y_{0}(t)\right\|_{2}},
$$

where the symbol $\|\cdot\|_{2}$ stands for the $L_{2}$ norm with the period $2 \pi / \omega$ and $\tilde{y}_{0}(t)$ denotes the steady-state periodic output of the system [26]. But, it is too difficult to calculate the undistorted signal $\tilde{y}_{0}(t)$ in the fractional-order system. As suggested in [16], the limits $\lim _{t \rightarrow \infty} y_{0}(t)$ and $\lim _{t \rightarrow \infty} \tilde{y}_{0}(t)$ are used to replace $y_{0}(t)$ and $\tilde{y}_{0}(t)$. Based on the final value theorem of Laplace transform, it follows

$$
\begin{aligned}
\lim _{t \rightarrow \infty} y_{0}(t) & =\lim _{s \rightarrow 0} s \mathscr{L}\left(y_{0}(t)\right) \\
& =\lim _{s \rightarrow 0} s\left(\frac{A_{0}}{s}+\frac{B_{0} \omega}{s^{2}+\omega^{2}}\right) \\
& =A_{0}, \\
\lim _{t \rightarrow \infty} \tilde{y}_{0}(t) & =\lim _{s \rightarrow 0} s \mathscr{L}\left(y_{0}(t)^{2}\right) G(s) \\
& =\lim _{s \rightarrow 0} s\left(\frac{A_{0}^{2}}{s}+\frac{2 A_{0} B_{0} \omega}{s^{2}+\omega^{2}}+\frac{2 B_{0}^{2} \omega^{2}}{s^{3}+4 s \omega^{2}}\right) G(s) \\
& =\frac{2 A_{0}^{2}+B_{0}^{2}}{2 a+2 b} .
\end{aligned}
$$

Substituting relations (32) and (33) into (31), the distortion index $\Delta$ can be obtained for different values $a, b$, and $q$. Indeed, small value of $\Delta$ implies that the predicted limit cycle is reliable. 


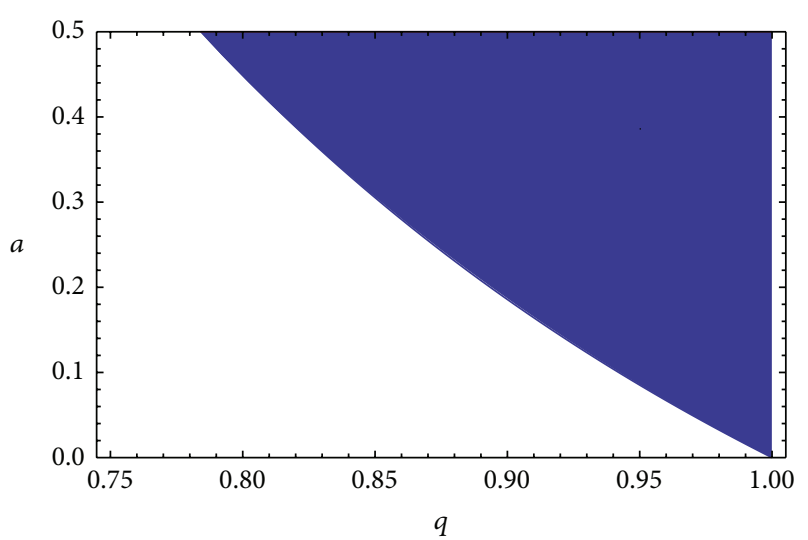

Figure 2: Possible chaotic parameter region for fractional-order Rössler toroidal system with $b=0.2$.

TABLE 1: Distortion indices $\Delta$ for different values of the parameter $a$.

\begin{tabular}{cccccc}
\hline$a$ & 0.1 & 0.2 & 0.3 & 0.4 & 0.5 \\
$\Delta$ & 9 & 4 & 2.3333 & 1.5 & 1 \\
\hline
\end{tabular}

\section{Numerical Simulations}

By fixing $b=0.2$, the possible chaotic parameter region for fractional-order Rössler toroidal system based on the Genesio-Tesi conjecture in the $q-a$ plane is shown in Figure 2. In the shaded region of Figure 2, it is possible for chaos to occur.

Next consider the filter effect for different parameter values. Based on the above expressions (31)-(33), an interesting thing has been found that different values $b$ and $q$ cannot affect the value of distortion index $\Delta$. Moreover, the values of distortion index $\Delta$ for different values $a$ are shown in Table 1.

According to Table 1, the filtering effect of the system decreases by increasing $a$. It means that the possibility of existence of chaos increases by choosing larger $a$ in the possible chaotic region (shown in Figure 2). To confirm the existence of chaotic behavior in fractional-order Rössler toroidal system (9), numerical simulations are carried out by applying the numerical method in Section 2.2. For instance, the pair $(q, a)=(0.95,0.386)$ is selected as a paradigm to confirm the existence of chaos (shown in Figure 3 ).

\section{Conclusions}

In this paper, harmonic balance based method is applied to detect the conditions indicating a chaotic behavior of fractional-order Rössler toroidal system. Based on the four steps of Genesio-Tesi conjecture, the possible chaotic parameter region is obtained. Also, numerical simulations are carried out to verify the accuracy and effectiveness of the obtained results. Finally, the finding in present paper indicates that harmonic balance method can give a useful approach to find rather simple conditions indicating a chaotic behavior of nonlinear fractional-order dynamical systems.

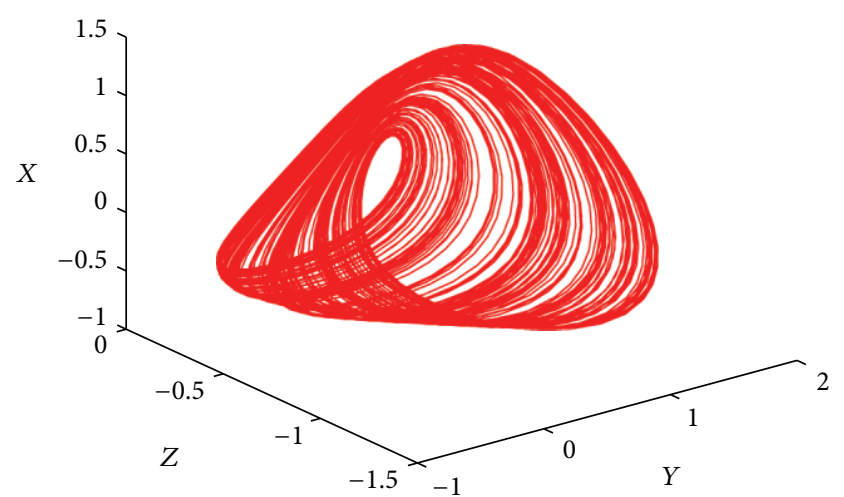

FIGURE 3: Chaotic attractor of fractional-order Rössler toroidal system with $(a, b, q)=(0.386,0.2,0.95)$.

\section{Acknowledgment}

This work was supported by the Science and Technology Innovation Projects of Education Bureau of Guangdong Province (no. 2012KJCX0073).

\section{References}

[1] K. B. Oldham and J. Spanier, The Fractional Calculus, Academic Press, London, UK, 1974.

[2] I. Podlubny, Fractional Differential Equations, vol. 198, Academic Press Inc., San Diego, Calif, USA, 1999.

[3] R. Hilfer, Applications of Fractional Calculus in Physics, World Scientific, River Edge, NJ, USA, 2000.

[4] T. T. Hartley, C. F. Lorenzo, and H. K. Qammer, "Chaos in a fractional order Chua's system," IEEE Transactions on Circuits and Systems I, vol. 42, no. 8, pp. 485-490, 1995.

[5] P. Arena, R. Caponetto, L. Fortuna, and D. Porto, "Chaos in a fractional order Duffing system," in Proceedings of European Conference on Circuit Theory and Design, pp. 1259-1262, Budapest, Hungary, 1997.

[6] I. Grigorenko and E. Grigorenko, "Chaotic dynamics of the fractional Lorenz system," Physical Review Letters, vol. 91, no. 3, Article ID 034101, 2003.

[7] C. G. Li and G. R. Chen, "Chaos and hyperchaos in the fractional-order Rössler equations," Physica A, vol. 341, no. 14, pp. 55-61, 2004.

[8] C. P. Li and G. R. Chen, "Chaos in the fractional order Chen system and its control," Chaos, Solitons and Fractals, vol. 22, no. 3, pp. 549-554, 2004.

[9] V. Daftardar-Gejji and S. Bhalekar, "Chaos in fractional ordered Liu system," Computers \& Mathematics with Applications, vol. 59, no. 3, pp. 1117-1127, 2010.

[10] Q. Yang and C. Zeng, "Chaos in fractional conjugate Lorenz system and its scaling attractors," Communications in Nonlinear Science and Numerical Simulation, vol. 15, no. 12, pp. 4041-4051, 2010.

[11] C. Zeng, Q. Yang, and J. Wang, "Chaos and mixed synchronization of a new fractional-order system with one saddle and two stable node-foci," Nonlinear Dynamics, vol. 65, no. 4, pp. 457466, 2011. 
[12] Z. Wei, "Synchronization of coupled nonidentical fractionalorder hyperchaotic systems," Discrete Dynamics in Nature and Society, vol. 2011, Article ID 430724, 9 pages, 2011.

[13] S. J. Linz, "No-chaos criteria for certain classes of driven nonlinear oscillators," Acta Physica Polonica B, vol. 34, no. 7, pp. 3741-3749, 2003.

[14] J. Guckenheimer and P. Holmes, Nonlinear Oscillations, Dynamical Systems, and Bifurcations of Vector Fields, vol. 42, Springer, New York, NY, USA, 1983.

[15] C. P. Silva, "Shil'nikov's theorem-a tutorial," IEEE Transactions on Circuits and Systems I, vol. 40, no. 10, pp. 675-682, 1993.

[16] Z. M. Wu, J. G. Lu, and J. Y. Xie, "Analysing chaos in fractionalorder systems with the harmonic balance method," Chinese Physics, vol. 15, no. 6, pp. 1201-1207, 2006.

[17] M. S. Tavazoei and M. Haeri, "Describing function based methods for predicting chaos in a class of fractional order differential equations," Nonlinear Dynamics, vol. 57, no. 3, pp. 363-373, 2009.

[18] K. Diethelm and N. J. Ford, "Analysis of fractional differential equations," Journal of Mathematical Analysis and Applications, vol. 265, no. 2, pp. 229-248, 2002.

[19] K. Diethelm, N. J. Ford, and A. D. Freed, "A predictor-corrector approach for the numerical solution of fractional differential equations," Nonlinear Dynamics, vol. 29, no. 1-4, pp. 3-22, 2002.

[20] O. E. Rössler, "Continuous chaos-four prototype equations," Annals of the New York Academy of Sciences, vol. 316, pp. 376392, 1979.

[21] K. Ciesielski, "On the Poincare-Bendixson theorem," in Lecture Notes in Nonlinear Analysis, vol. 3, pp. 49-69, 2002.

[22] R. Genesio and A. Tesi, "Harmonic balance methods for the analysis of chaotic dynamics in nonlinear systems," Automatica, vol. 28, no. 3, pp. 531-548, 1992.

[23] A. Gelb and W. E. Velde, Multiple-Input Describing Functions and Nonlinear System Design, McGraw Hill, New York, NY, USA, 1967.

[24] F. A. Savaci and S. Günel, "Harmonic balance analysis of the generalized Chua's circuit," International Journal of Bifurcation and Chaos in Applied Sciences and Engineering, vol. 16, no. 8, pp. 2325-2332, 2006.

[25] C. Bonnet and J. R. Partington, "Coprime factorizations and stability of fractional differential systems," Systems \& Control Letters, vol. 41, no. 3, pp. 167-174, 2000.

[26] R. Genesio, A. Tesi, and F. Villoresi, "Frequency approach for analyzing and controlling chaos in nonlinear circuits," IEEE Transactions on Circuits and Systems I, vol. 40, no. 11, pp. 819828, 1993. 


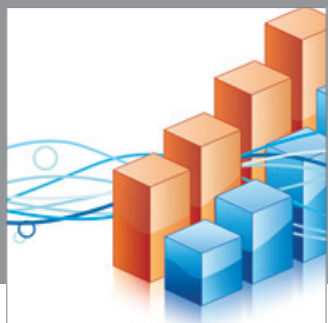

Advances in

Operations Research

mansans

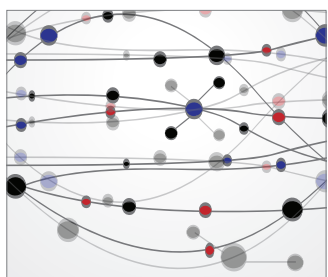

The Scientific World Journal
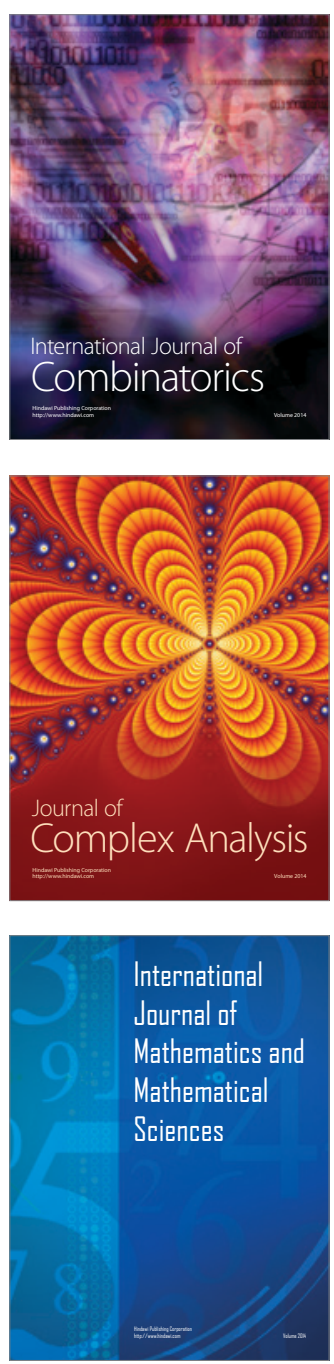
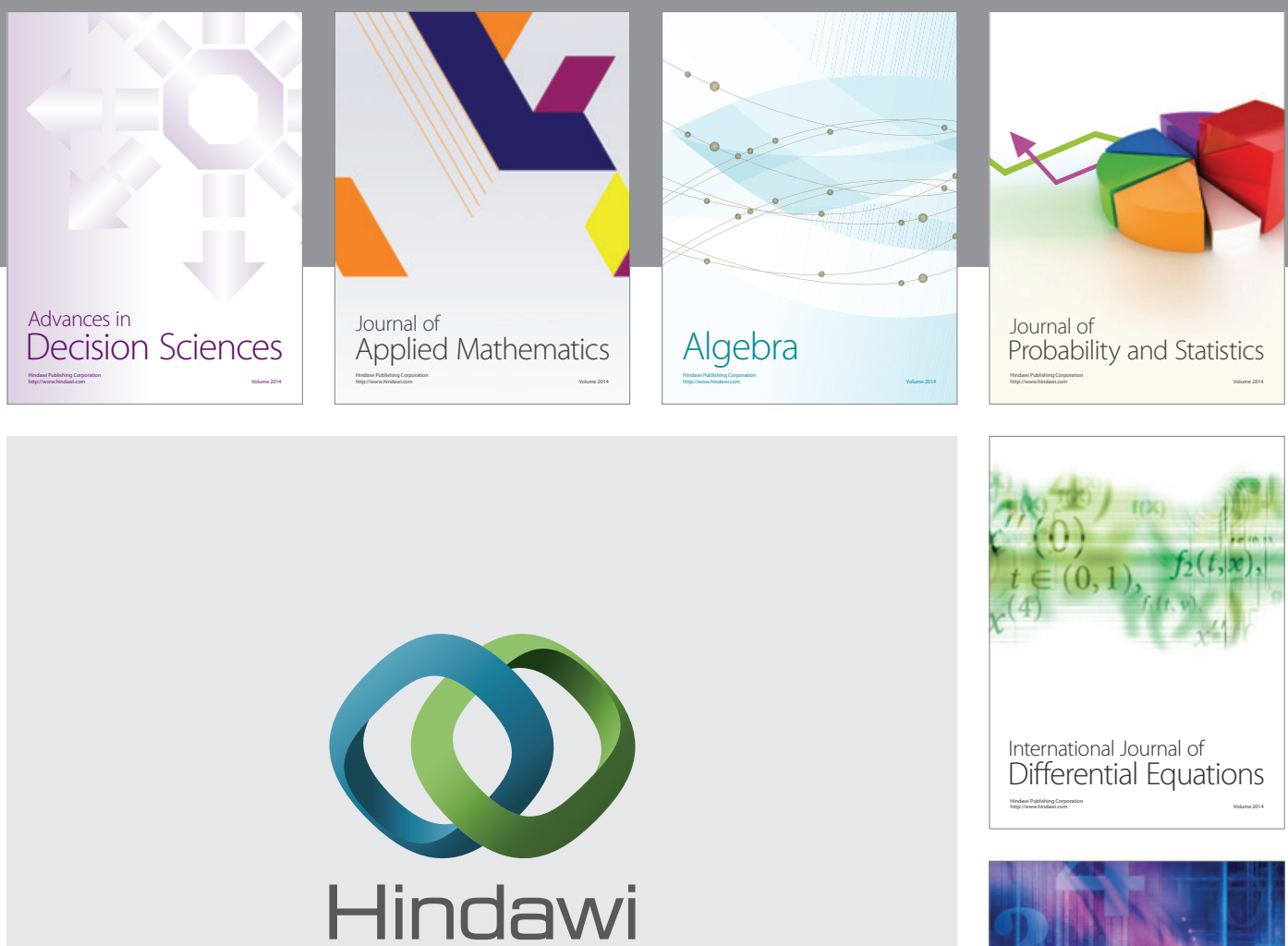

Submit your manuscripts at http://www.hindawi.com
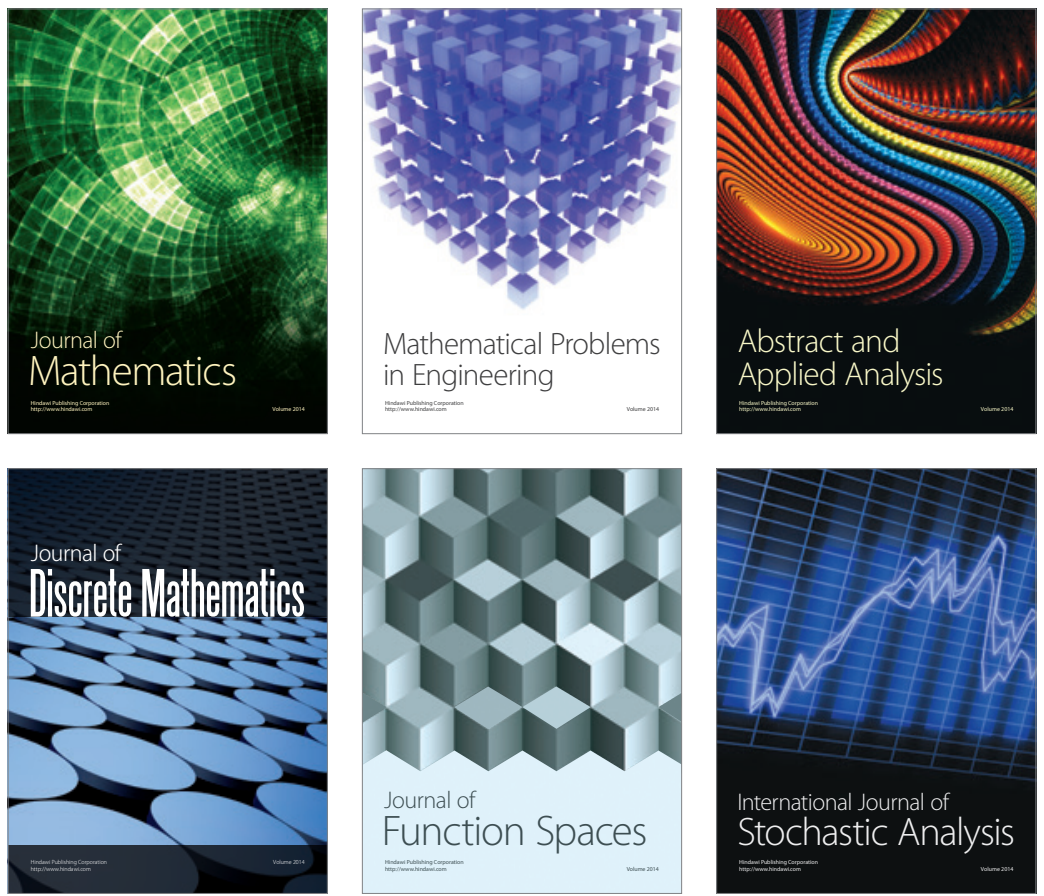

Journal of

Function Spaces

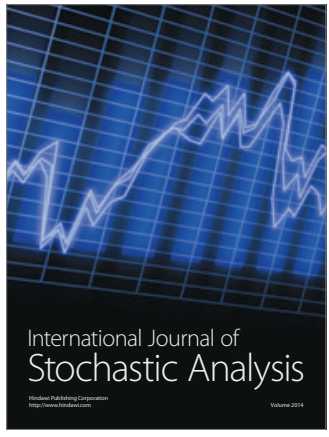

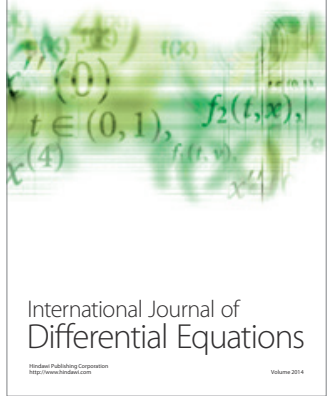
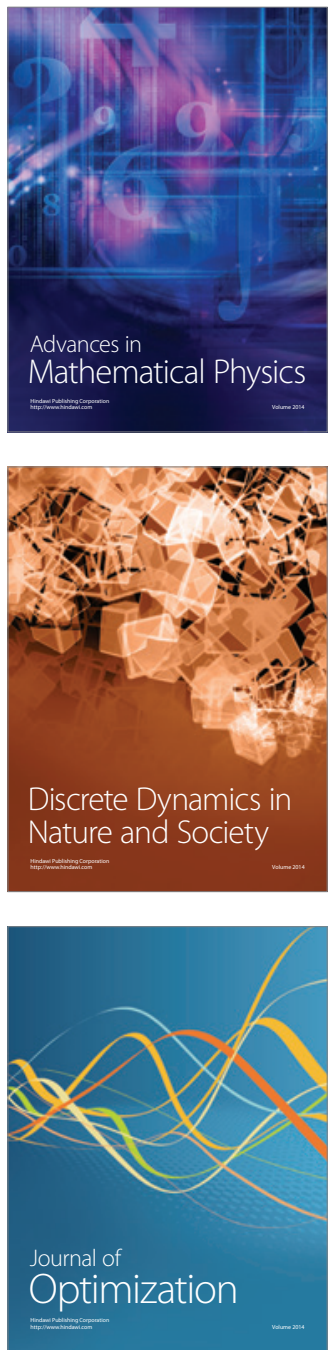